\title{
A plicabilidade do sistema de proteção contra descargas atmosféricas com foco na segurança de imóveis e seres vivos
}

\author{
Applicability of lightning protection system as focus on the safety of real estate and living beings
}

\author{
Alex Franco Ferreira*, Célio de Souza Sobreira
}

Como citar esse artigo. Ferreira, AF; Sobreira, CS. Aplicabilidade do sistema de proteção contra descargas atmosféricas com foco na Segurança ne Imóveis e Seres Vivos. Revista Teccen. 2019 Jul/Dez; 12 (2): 75-82.

\begin{abstract}
Resumo
Devido à grande quantidade de descargas atmosféricas que ocorrem no Brasil, faz-se necessário a instalação de um sistema de proteção contra descargas atmosféricas para que se possam proteger estruturas ou seres vivos que residam ou estejam momentaneamente no interior de imóveis, estruturas ou em suas imediações. Contudo, para implementação de um sistema de proteção contra descargas atmosféricas é preciso que se faça uma avaliação dos riscos que uma estrutura possa apresentar levando em conta o tipo de estrutura, as perdas que essa estrutura pode ocasionar. Para avaliar esses riscos é feito um gerenciamento que tem como finalidadede verificar se uma estrutura necessita ou não de implementação de um sistema de proteção contra descargas atmosféricas, conhecido como SPDA. Contudo, para a segurança,sempre se deve levar em consideração que mesmo que uma avaliação de risco apresente um resultado no qual não necessite de um sistema de proteção contra descargas atmosféricas, à decisão de implementação pode ser independente do resultado da análise de risco.
\end{abstract}

Palavras-Chave: Proteção, SPDA, Segurança.

\begin{abstract}
Due to the large amount of atmospheric discharges that occur in Brazil, it is necessary to install a system to protect against these discharges so that protect structures or living beings that live in the buildings or in their surroundings. However, to implement a protection system against atmospheric discharges, it is necessary to measure the risks in order that structures can present, consideringthe type of structures and the damages that it can cause.To calculate these risks is done a management of them to measure whether a structure needs to implement a lightning protection system known as SPDA.Although, for safety, it should always been observed that even if a risk assessment presents a result in which it does not require a protection against lightning, the implementation decision may be independent of the results of the risk analysis.
\end{abstract}

Keywords: Protection, SPDA, Security.

\section{Introdução}

De acordo com Mamede (2017), as descargas atmosféricas criam sério a perturbações nas redes aéreas de transmissão e distribuição de energia elétrica, causando sérios danos materiais nas construções atingidas por essas descargas, sem deixar de levar em consideração o risco de morte a que as pessoas e animais estão expostos.

O Brasil é um dos países com a maior incidência de descargas atmosféricas do planeta, segundo Inpe (2017), devido a esse fator um sistema de proteção contra descargas atmosféricas é de grande importância. No Brasil a Norma Parte 2 da ABNT NBR 5419 (2015), Proteção de estruturas contra descargas atmosféricas, é responsável por determinar as diretrizes para viabilidade de um SPDA, está norma impõe as condições de projeto e instalação de um sistema SPDA, com foco em proteger as estruturas contra a incidência direta de descargas atmosféricas. A proteção é aplicada também contra a incidência direta dos raios sobre os equipamentos e seres vivosque se encontrem no interior destas estruturas ou no interior da área de proteção imposta pelo SPDA instalado, captando e fazendo o direcionando da corrente proveniente da descarga para sistemas de aterramento.

De acordo com Henriques (2015), a primeira finalidade desse sistema é neutralizar não só o poder de atração das pontas, mas também o crescimento do gradiente de potencial elétrico entre o solo e as nuvens, por meio do escoamento de cargas elétricas para a terra. A segunda finalidade é fornecer à descarga elétrica um caminho para escoar, de baixa impedância, mitigando os riscos provenientes da sua incidência. 
Este trabalho tem por finalidade apresentar a Análise de risco para determinação da necessidade da implementação de um Sistema de Proteção Contra descargas atmosféricas em uma construção localizada do município de Vassouras no estado do Rio de Janeiro em prol da segurança do imóvel e dos seres vivos que habitam o local.

Para viabilidade de um sistema de proteção contra descargas atmosféricas, segundo a norma ABNT NBR 5419-2 (2015), onde foi feito primeiramente um gerenciamento de risco, sendo levantados diversos parâmetros para os cálculos necessários para avaliar a necessidade de implantação de um sistema de proteção em uma estrutura. Todos os passos para execução deste estudo basearam-se na sequência metodológica apresentada na ABNT NBR 5419-2 (2015), onde no estudo de gerenciamento de risco foram levantados alguns procedimentos básicos como a verificação da incidência de descargas atmosféricas da região, o ponto de impacto de uma descarga atmosférica, a identificação do tipo de dano causada pela estrutura, o tipo de perdas que a estrutura pode causar, o tipo de risco onde sãoanalisadas as componentes para cada tipo de perda e por fim a comparação dos riscos pelos riscos toleráveis.

\section{Sistema de proteção contra descarga atmosférica}

Para instalar um sistema de proteção contra descarga atmosférica deve-se fazer primeiramente um gerenciamento de risco, onde são analisados os fatores necessários que impactam na viabilidade de um sistema de proteção contra descarga atmosférica.

Segundo a norma ABNT NBR 5419-2 (2015), os seguintes itens devem ser considerados para o gerenciamento de risco deve ser analisado o próprio tipo de estrutura que são classificadas como fontes dos danos onde é encontrado o tipo de influência da descarga nas estruturas, essas fontes dos danos são diferentes em decorrência do ponto de impacto da descarga podendo ser classificadas como descarga atmosférica na estrutura (S1), descarga atmosférica próximo da estrutura (S2), descarga atmosférica na linha de transmissão (S3) e descarga atmosférica próximo da linha de transmissão (S4), logo em seguida de ser levado em consideração a possível fontes de danosdevendo analisar os tipos de danos que essas fontes podem causar, sendo esses danos divididos em três categorias: danos a seres humanos em consequência de choques elétricos (D1), danos físicos em consequência dos efeitos das correntes das descargas atmosféricas (D2) e falhas de sistemas internos em consequência de pulsos eletromagnéticos (D3), depois de analisado o tipo de dano deve-se analisar qual tipo de perda que os danos podem gerar, sendo essas perdas divididas em quatro tipos: L1 perda de vida humana (incluindo ferimentos), L2 perda de serviço ao público, L3 perda de patrimônio cultural e L4 perda de valor econômico, para cada tipo de perda (L),logo depois de analisado essas perdas deve-se passar para o cálculo do Risco (R), o qual depende de componentes mencionados anteriormente podendo ser verificado conforme segue Quadro 1.

Quadro 1. Fontes de Danos, Tipos de Danos e Tipos de Perdas de acordo com o Ponto de impacto.

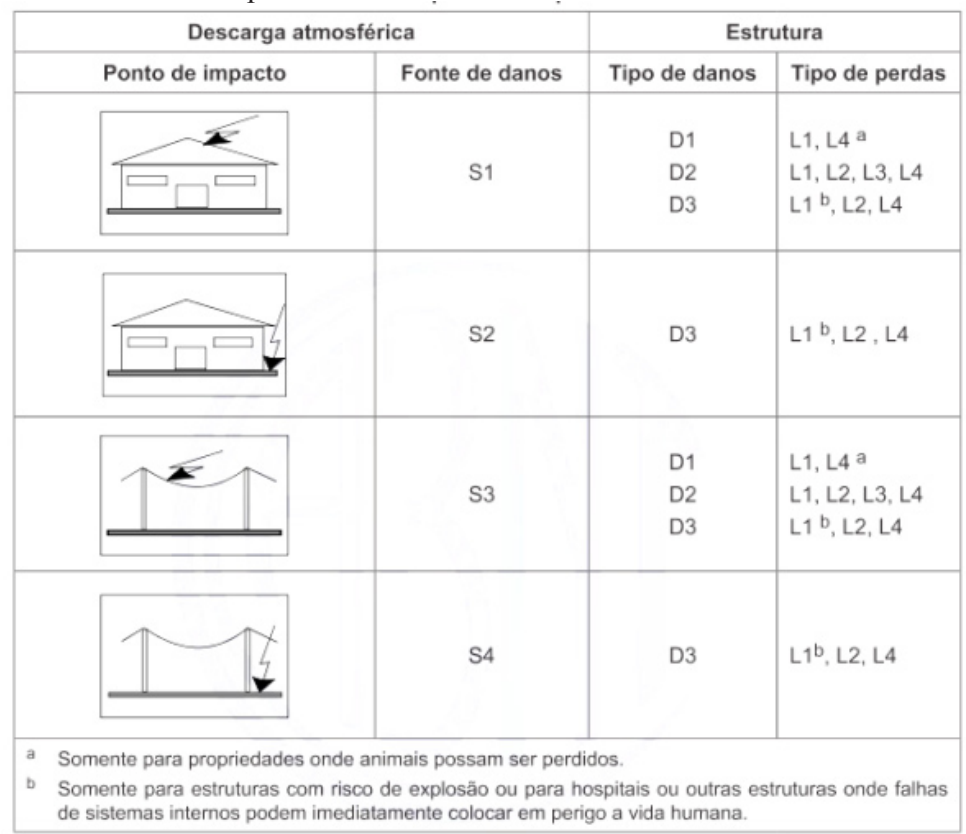

Fonte: ABNT NBR 5419-2 (2015). 
De acordo com o Portal O setor elétrico (2015) o risco é definido como o valor de uma possível perda média anual em consequência de descargas atmosféricas, em relação ao valor total do objeto a ser protegido. Os riscos decorrem da quantidade de descargas atmosféricas anuais que cai em uma estrutura, de um possível dano causado por uma das descargas atmosféricas que influenciam esta estrutura e da quantidade média das perdas causadas.

A norma ABNT NBR 5419-2 (2015) avalia os riscos em uma estrutura da seguinte forma: R1 - risco de perda de vida humana, R2 - risco de perda de instalação de serviço ao público, R3 - risco de perda de memória cultural e R4 - risco de perda de valor econômico.

Para uma dada perda (L) existirá um Risco (R) a ser calculado, o qual depende de componentes que são agrupados de acordo com a fonte de danos (S) e o tipo de dano (D), conforme segue abaixo.

Segundo Souza (2016) para iniciar um cálculo de um determinado risco, sãousados diversos tipos de componentes de risco que devem ser avaliados:

\section{Componentes de risco para uma estrutura devido às descargas nas estruturas de acordo com a ABNT NBR 5419-2 (2015)}

RA - Componente relativo ao tipo de ferimentos causados aos seres vivos por meio de choque elétrico provocado por tensões de passo e de toque em distâncias de até $3 \mathrm{~m}$, na parte externa da estrutura, nessa componente deve-se levar em consideração a perda L1 em casos em que a estrutura tenha animais vivos e também as perdas L4 onde pode aumentar as perdas de animais vivos;

RB - Componente relativo a anomalias físicas geradas por centelhamentos perigosos na parte interna da estrutura levando a um incêndio ou explosão, podendo também levar perigo ao meio ambiente, nesse componente é usado todos os tipos de perdas (L1,L2,L3 e L4).

RC - Componente criado pela falha de sistemas internos gerados por LEMPs (pulsos eletromagnéticos devido aos raios) em consequência de uma descarga que venha a cair na estrutura, nessa componente as perdas L2 e L4 pode vir a acontecer em todos os casos juntamente com a perda L1, nos casos de estruturas com risco de explosão e hospitais ou em outras estruturas de modo que falhas de sistemas internos coloquem em perigo a vida humana.

\section{Componentes de risco para uma estrutura devido às descargas perto da estrutura de acordo com a ABNT NBR 5419-2 (2015)}

RM - Falha de sistemas internos criada por LEMP em consequência de uma descarga que venha a cair em áreas perto da estrutura, nessa componente as perdas L2 e L4 pode vir a acontecer em todos os casos juntamente com a perda L1, nos casos de estruturas com risco de explosão e hospitais ou em outras estruturas de modo que falhas de sistemas internos coloquem em perigo a vida humana;

\section{Componentes de risco para uma estrutura devido às descargas a uma linha conectada à estruturade acordo com a ABNT NBR 5419-2 (2015)}

RU - Componente relativo a ferimentos gerados em seres vivos por meio de choque elétrico em consequência das tensões de toque e passo no interior da estrutura criada por descargas que atingiram a linha elétrica ligada a estrutura, nessa componente a perda L1 pode ocorrer no caso de propriedades agrícolas, e as perdas L4 em consequência de prováveis perdas de animais;

RV - Componente relativo a anomalias físicas (incêndio ou explosão iniciados por centelhamentos perigosos entre instalações externas e partes metálicas geralmente no ponto de entrada da linha na estrutura) em consequência da descarga atmosférica e correntetransmitida ao longo das linhas, nessa componente todos as perdas (L1,L2,L3 e L4) podem acontecer;

RW - Componente relativo a falhas de sistemas internos criados por sobretensões que são induzidas nas linhas que entram na estrutura e transmitidas a esta, nessas componentes podem ocorrer a perdas L2 e L4 em todos os casos, juntamente com a perda L1nos casos de estruturas com risco de explosão e hospitais ou em outras estruturas de modo que falhas de sistemas internos coloquem em perigo a vida humana;

\section{Componentes de risco para uma estrutura devido às descargas perto de uma linha conectada à estrutura de acordo com a ABNT NBR 5419-2 (2015)}

RZ - Componente relativo a falhas de sistemas internos criados por sobretensões que são induzidas nas linhas que entram na estrutura e transmitidas a esta, nessas componentes podem ocorrer a perdas L2 e L4 em todos os casos, juntamente com a perda L1 nos 
casos de estruturas com risco de explosão e hospitais ou em outras estruturas de modo que falhas de sistemas internos coloquem em perigo a vida humana;

\section{Equações de cada componentes de risco para uma estrutura devido às descargas}

Segundo Sueta (2015), para obter o valor de cada componenteutiliza-se a Equação 1.0.

$$
\mathrm{R}_{\mathrm{x}}=\mathrm{N}_{\mathrm{x}} \times \mathrm{P}_{\mathrm{x}} \times \mathrm{L}_{\mathrm{x}}, \quad(1.0)
$$

Onde, $\mathrm{N}_{\mathrm{x}}=$ é o número de ventos perigosos por ano; $\mathrm{P}_{\mathrm{x}}=$ é a probabilidade de dano à estrutura; $\mathrm{L}_{\mathrm{x}}=$ é $\mathrm{a}$ perda consequente;

De acordo com Souza (2016) depois de apresentados todos os componentes de risco, as relações matemáticas correspondentes à composição de cada um deles podem ser explicitadas baseando-se nos seguintes pontos:

- Análise dos cálculos dos componentes de risco devido a descargas atmosféricas em uma estrutura (S1), com o componente referente a ferimentos a seres vivos em função de choque elétrico (D1), com o componente referente a anomalias físicas (D2) e com o componente referente à falha de sistemas na parte interna da estrutura (D3) usa-se as equações que se encontrano Quadro 2.

- Análise dos cálculos dos componentes de risco devido a descargas atmosféricas perto da estrutura (S2), com o componente referente à falha do sistema interno (D3) usa-se as equações que se encontra no Quadro 2.

- Análise dos cálculos dos componentes de risco devido a descargas atmosféricas em uma linha conectada a uma estrutura (S3), com componente referente a ferimentos causados em seres vivos em consequência de choque elétrico (D1), com o componente referente a anomalias físicas (D2) e como à componente referente à falha do sistema interno (D3) usa-se as equações que se encontra no Quadro 2.

- Análise dos cálculos dos componentes de risco devido a descargas atmosféricas perto de uma linha conectada a uma estrutura (S4), com componente referente à falha dos sistemas internos (D3) usa-se as equações que estão localizadas Quadro 2.

Quadro 2. Componentes de risco para diferentes tipos de danos e fontes de danos

\begin{tabular}{|c|c|c|c|c|}
\hline \multirow[b]{2}{*}{ Danos } & \multicolumn{4}{|c|}{ Fonte de danos } \\
\hline & $\begin{array}{c}\text { Sl } \\
\text { Descarga } \\
\text { Atmosférica } \\
\text { na estrutura }\end{array}$ & $\begin{array}{c}\text { S2 } \\
\text { Descarga } \\
\text { atmosférica perto } \\
\text { da estrutura }\end{array}$ & $\begin{array}{c}\mathrm{S3} \\
\text { Descarga } \\
\text { atmosférica na } \\
\text { linha conectada }\end{array}$ & $\begin{array}{c}\text { S4 } \\
\text { Descarga } \\
\text { Atmosférica } \\
\text { perto da linha } \\
\text { conectada }\end{array}$ \\
\hline $\begin{array}{c}\text { D1 } \\
\text { Ferimentos a seres } \\
\text { vivos devido a } \\
\text { choque elétrico }\end{array}$ & $\begin{array}{r}\mathrm{R}_{\mathrm{A}}=\mathrm{N}_{\mathrm{D}} \times \mathrm{P}_{\mathrm{A}} \\
\times \mathrm{L}_{\mathrm{A}}\end{array}$ & & $\begin{aligned} R_{U}= & \left(N_{L}+N_{D J}\right) \\
& x P_{U} \times L_{U}\end{aligned}$ & \\
\hline $\begin{array}{c}\text { D2 } \\
\text { Danos fisicos }\end{array}$ & $\begin{array}{r}\mathrm{R}_{B}=\mathrm{N}_{D} \times \mathrm{P}_{B} \\
\times \mathrm{L}_{B}\end{array}$ & & $\begin{array}{c}\mathrm{R}_{\mathrm{V}}=\left(\mathrm{N}_{\mathrm{L}}+\mathrm{N}_{\mathrm{DJ}}\right) \\
\mathrm{x} \mathrm{P}_{\mathrm{V}} \mathrm{x} \mathrm{L}_{\mathrm{V}}\end{array}$ & \\
\hline $\begin{array}{l}\text { D3 } \\
\text { Falha de sistemas } \\
\text { eletroeletrônicos }\end{array}$ & $\begin{array}{r}\mathrm{R}_{C}=\mathrm{N}_{\mathrm{D}} \times \mathrm{P}_{\mathrm{C}} \\
\times \mathrm{L}_{\mathrm{C}}\end{array}$ & $\begin{array}{c}\mathrm{R}_{\mathrm{M}}=\mathrm{N}_{\mathrm{M}} \times \mathrm{P}_{\mathrm{M}} \\
\times \mathrm{L}_{\mathrm{M}}\end{array}$ & $\begin{array}{r}R_{W}=\left(N_{L}+N_{D J}\right) \\
\quad \times P_{W} \times L_{W}\end{array}$ & $\begin{array}{r}\mathrm{R}_{Z}=\mathrm{N}_{\mathrm{I}} \times \mathrm{P}_{\mathrm{Z}} \\
\times \mathrm{L}_{Z}\end{array}$ \\
\hline
\end{tabular}

Fonte: ABNT NBR 5419-2 (2015)

De acordo comFergütz (2015), os parâmetros $\mathrm{N}$ são encontrados no Anexo $\mathrm{A}$, os parâmetros $\mathrm{P}$ são encontrados no Anexo $\mathrm{B}$ e os parâmetros L são encontrados no Anexo C, da norma 5419/2015. Nestes Anexos, encontram-se as fórmulas para os cálculos dos parâmetros e tabelas com valores a serem utilizados nos cálculos.

De acordo com a norma Parte 2 da ABNT NBR
5419 (2015), o cálculo dos riscos é feito pela somatória de alguns componentes, de acordo com as seguintes equações:

R1: Risco de perda de vida humana, de acordo com a equação 2.0 :

$\mathrm{R} 1=\mathrm{R}_{\mathrm{A} 1}+\mathrm{R}_{\mathrm{B} 1}+\mathrm{R}_{\mathrm{C} 1}{ }^{1}+\mathrm{R}_{\mathrm{M} 1}{ }^{1}+\mathrm{R}_{\mathrm{V} 1}+\mathrm{R}_{\mathrm{w} 1}{ }^{1}+\mathrm{R}_{\mathrm{Z} 1}{ }^{1}$

1 - Somente para estrutura com risco de explosão 
Quadro 4. Componentes de risco a serem considerados para cada tipo de perda em uma estrutura

\begin{tabular}{|c|c|c|c|c|c|c|c|c|}
\hline \multirow{2}{*}{$\begin{array}{l}\text { Fontes de danos } \\
\\
\begin{array}{c}\text { Componente de } \\
\text { risco }\end{array}\end{array}$} & \multicolumn{3}{|c|}{$\begin{array}{c}\text { Descarga } \\
\text { Atmosférica } \\
\text { na estrutura } \\
\text { Sl }\end{array}$} & \multirow{2}{*}{$\begin{array}{c}\begin{array}{c}\text { Descarga } \\
\text { atmosférica } \\
\text { perto }\end{array} \\
\text { da estrutura } \\
\mathrm{S} 2\end{array}$} & \multicolumn{3}{|c|}{$\begin{array}{c}\text { Descarga } \\
\text { atmosférica em uma } \\
\text { linha conectada } \\
\text { à estrutura } \\
\text { S3 }\end{array}$} & \multirow{2}{*}{ 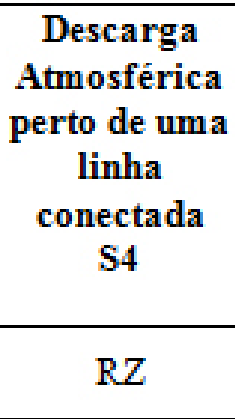 } \\
\hline & $\mathrm{R}_{\mathrm{A}}$ & $\mathrm{R}_{B}$ & $\mathrm{R}_{\mathrm{C}}$ & & $\mathrm{R}_{\mathrm{U}}$ & $\mathrm{R}_{\mathrm{V}}$ & $\mathrm{R}_{\mathrm{N}}$ & \\
\hline \multicolumn{9}{|l|}{$\begin{array}{l}\text { Risco para cada } \\
\text { tipo de perda }\end{array}$} \\
\hline R1 & $*$ & $*$ & $* a$ & $* a$ & $*$ & $*$ & $* a$ & $* a$ \\
\hline R2 & & * & $*$ & $*$ & & $*$ & $*$ & $*$ \\
\hline R3 & & $*$ & & & & $*$ & & \\
\hline R4 & ${ }^{* b} \mathrm{~b}$ & * & * & $*$ & *b & $*$ & $*$ & $*$ \\
\hline
\end{tabular}

e para hospitais com equipamentos elétricos para salvar vidas ou outras estruturas quando à falha dos sistemas internos imediatamente possa colocar em perigo a vida humana.

R2: Risco de perda de serviço ao público de acordo com a equação 3.0.

$$
\mathrm{R} 2=\mathrm{R}_{\mathrm{B} 2}+\mathrm{R}_{\mathrm{C} 2}+\mathrm{R}_{\mathrm{M} 2}+\mathrm{R}_{\mathrm{V} 2}+\mathrm{R}_{\mathrm{w} 2}+\mathrm{R}_{\mathrm{Z} 2}
$$

R3: Risco de perda de patrimônio cultural de acordo com a equação 4.0.

$$
\mathrm{R} 3=\mathrm{R}_{\mathrm{B} 3}+\mathrm{R}_{\mathrm{V} 3}
$$

R4: Risco de perda de valor econômico de acordo com a equação 5.0.

$\mathrm{R} 4=\mathrm{R}_{\mathrm{A} 4}{ }^{2}+\mathrm{R}_{\mathrm{B} 4}+\mathrm{R}_{\mathrm{C} 4}+\mathrm{R}_{\mathrm{M} 4}+\mathrm{R}_{\mathrm{U} 4}{ }^{2}+\mathrm{R}_{\mathrm{V} 4}+\mathrm{R}_{\mathrm{W} 4}+\mathrm{R}_{\mathrm{Z} 4}$

2 - Somente para propriedades onde possam ser perdidos animais

De acordo com Portal o setor elétrico (2015), a partir do momento em que se calcula o risco, os valores de cada risco são comparados aos valores toleráveis, onde a norma determina os limites para cada tipo de risco, chamado de Risco Tolerável (RT), de acordo com cada tipo de perda envolvida, como segue Quadro 3:

$\mathrm{Se} \mathrm{R} \leq \mathrm{RT}$, não é necessária a proteção contra descargas atmosféricas;

Se R > RT, então medidas devem ser tomadas para se obter $\mathrm{R} \leq \mathrm{RT}$.
Estudo de Caso: Análise de risco para determinação da necessidade da implementação de um Sistema de Proteção Contra descargas atmosféricas

Análise de gerenciamento de risco de um prédio com 5 apartamentos com um total de 10 pessoas em um território plano com estruturas menores na redondeza, tendo uma altura mínima $(\mathrm{Hm})$ de $11 \mathrm{~m}$, comprimento (L) de $15 \mathrm{~m}$ e largura (W) de $12 \mathrm{~m}$. Linhas de energia a $10 \mathrm{~m}$ sendo aérea e sem blindagem localizado em Vassouras -RJ.

\section{Considerações para a análise de risco}

Edificação residencial apresenta a possibilidade de perda de vida humana.

- Nessa situação deve-se somente calcular o risco R1 para perda de vida humana e perdas L1 com suas respectivas componentes $\mathrm{RA}, \mathrm{RB}, \mathrm{RU}, \mathrm{e} \mathrm{RV}$, conforme o Quadro 4:

- Risco tolerável (RT) para perda L1 será de 10-5 de acordo com o Quadro 3.

- Riscos devido às descargas atmosféricas na estrutura (S1). 


\section{Análise das componentes de risco devido às descargas atmosférica na estrutura (S1)}

1- Componente relativo ao tipo de ferimentos causados aos seres vivos por meio de choque elétrico $\left(\mathrm{R}_{\mathrm{A}}\right)$, apresentado no Quadro 2.

A Equação 6.0 apresentada no anexo A da ABNT NBR 5419-2 (2015), indica o número de eventos perigosos para a estrutura $\mathrm{N}_{D}$.

$$
\mathrm{N}_{\mathrm{D}}=\mathrm{N}_{\mathrm{G}} \mathrm{X} \mathrm{A}_{\mathrm{D}} \mathrm{X} \mathrm{C}_{\mathrm{D}} \mathrm{X} \mathrm{10}^{-6}(6.0)
$$

De acordo com a norma ABNT NBR 5419-2 (2015). O fator de utilização da estrutura $\left(C_{D}\right)$ deve ser especificado através da Tabela A.1, a área de exposição equivalente $\mathrm{em}^{2}$ (vide cálculo abaixo) e a densidade de descargas atmosféricas para a terra em km²/ano $\left(\mathrm{N}_{\mathrm{G}}\right)$ deve ser estabelecido através da ELAT 2017.

$$
\mathrm{N}_{\mathrm{G}}=14,14 \mathrm{~km}^{2} / \mathrm{ano} ; \quad \mathrm{A}_{\mathrm{D}}=5383,19 \mathrm{~m}^{2}
$$
de $\mathrm{N}_{\mathrm{D}}$.

Resolvendo-se a Equação 6.0, obtém-se o valor

$$
\mathrm{N}_{\mathrm{D}}=0,5 \times 5383,19 \times 14,14 \times 10^{-6}=0,038
$$

A Equação 7.0 apresentada no anexo A da ABNT NBR 5419-2 (2015), indica aárea de exposição equivalente $A_{D}$ :

$\mathrm{A}_{\mathrm{D}}=\mathrm{LX} \mathrm{W}+2 \mathrm{X}(3 \mathrm{X} \mathrm{Hm}) \mathrm{X}(\mathrm{L}+\mathrm{W})+\pi \mathrm{X}(3 \mathrm{Hm})^{2}$

Para o prédio em questão tem se os seguintes parâmetros onde a altura mínima $(\mathrm{Hm})$ de $11 \mathrm{~m}$, comprimento (L) de $15 \mathrm{~m}$ e largura (W) de $12 \mathrm{~m}$.

$\mathrm{A}_{\mathrm{D}}=15 \mathrm{X} 12+2 \mathrm{X}(3 \mathrm{X} 11) \mathrm{X}(15+12)+\pi \mathrm{X}(3 \mathrm{X} 11)^{2}$ $=5383,19 \mathrm{~m}^{2}$

A Equação 8.0 apresentada no anexo A da ABNT NBR 5419-2 (2015), indica o cálculo de uma descarga atmosférica em uma estrutura causar ferimentos a seres vivos por meio de choque elétrico $\mathrm{P}_{\mathrm{A}}$.

$$
\mathrm{P}_{\mathrm{A}}=\mathrm{P}_{\mathrm{TA}} \mathrm{XP}_{\mathrm{B}}
$$

De acordo com a norma ABNT NBR 5419-2 (2015) o fator que depende do nível de proteção do SPDA $\left(\mathrm{P}_{\mathrm{B}}\right)$ deve ser especificado através da Tabela B.2 é o fator que depende das medidas de proteção adicionais contra tensões de toque e passo $\left(\mathrm{P}_{\mathrm{TA}}\right)$ deve ser especificado através da Tabela B.1.
Sendo $\mathrm{P}_{\mathrm{TA}}=1$ e $\mathrm{P}_{\mathrm{B}}=1$, obtém-se $\mathrm{P}_{\mathrm{A}}=1 \mathrm{X} 1$, então $\mathrm{P}_{\mathrm{A}}=1$.

A Equação 9.0 apresentada no anexo c da ABNT NBR 5419-2 (2015), indica os valores da perda $L_{A}$.

$$
\mathrm{L}_{\mathrm{A}}=\mathrm{r}_{\mathrm{t}} \mathrm{X} \mathrm{LT}_{\mathrm{T}} \mathrm{X}\left(\frac{n \mathrm{Z}}{n t}\right) \mathrm{X}\left(\mathrm{t}_{\mathrm{z}} / 8760\right)
$$

De acordo com a norma ABNT NBR 5419-2 (2015), o número relativo médio típico de vítimas feridas por choque elétrico devido a evento perigoso $\left(\mathrm{L}_{\mathrm{T}}\right)$ deve ser especificado através da Tabela C.2 é o fator de redução da perda de vida humana dependendo do solo/piso $\left(r_{t}\right)$ deve ser especificado através da Tabela C.3. O número de pessoas na zona $\left(\mathrm{n}_{\mathrm{z}}\right)$, o tempo durante o qual as pessoas estão presentes na zona expresso em $\mathrm{h} /$ ano $\left(\mathrm{t}_{\mathrm{z}}\right)$ e o número total de pessoas na estrutura $\left(\mathrm{n}_{\mathrm{t}}\right)$ são dados do projeto.

$$
\begin{array}{r}
r_{t}=10^{-3} ; L_{T}=10^{-2} ; n_{z}=10 ; n_{t}=10 ; t_{z}=8760 h / a n o \\
L_{A}=10^{-3} \times 10^{-2} \times\left(\frac{10}{10}\right) \times(8760 / 8760)=10^{-5}
\end{array}
$$

Assim, pode-se calcular o valor de $\mathrm{R}_{\mathrm{A}}$ :

$$
\mathbf{R}_{\mathbf{A}}=0,038 \times 1 \times 10^{-5}=0,038 \times 10^{-5}
$$

2 - Componente relativo ao tipo de ferimentos causados aos seres vivos por meio de choque elétrico $\left(\mathrm{R}_{\mathrm{B}}\right)$, apresentado no Quadro 2.

$\mathrm{O}$ valor de $\mathrm{N}_{\mathrm{D}}$, já foi determinado anteriormente: 0,038 .

$\mathrm{O}$ valor $\mathrm{P}_{\mathrm{B}}$ já foi determinado através da tabela 2 da norma ABNT NBR 5419-2 (2015):

A Equação 10 apresentada no anexo c da ABNT NBR 5419-2 (2015), indica os valores da perda $L_{B}$.

$$
L_{B}=r_{p} \times r_{f} \times h_{Z} \times L_{F} \times\left(\frac{n Z}{n t}\right) \times\left(t_{z} / 8760\right)
$$

De acordo com a norma ABNT NBR 5419-2 (2015), o fator de redução da perda a danos físicos depende das providências tomadas para reduzir as consequências do incêndio $\left(r_{p}\right)$ deve ser especificado através da Tabela C.4, o fator de redução da perda devido a danos físicos dependendo do risco de incêndio ou explosão da estrutura $\left(r_{f}\right)$ deve ser especificado através da Tabela C.5, o fator de aumento da perda devido a danos físicos quando um perigo especial não estiver presente $\left(h_{z}\right)$ deve ser especificado através da Tabela C.6, o número relativo médio típico de vítimas 
feridas por choque elétrico devido a evento perigoso $\left(\mathrm{L}_{\mathrm{F}}\right)$ deve ser especificado através da Tabela C.2. O número de pessoas na zona $\left(\mathrm{n}_{\mathrm{z}}\right)$, o tempo durante o qual as pessoas estão presentes na zona expresso em h/ano $\left(\mathrm{t}_{\mathrm{z}}\right)$ e o número total de pessoas na estrutura $\left(\mathrm{n}_{\mathrm{t}}\right)$ são dados do projeto.

$$
\begin{aligned}
& \quad \begin{array}{r}
\mathrm{r}_{\mathrm{p}}=0,5 ; \mathrm{r}_{\mathrm{f}}=10^{-3} ; \mathrm{h}_{\mathrm{z}}=1 ; \mathrm{L}_{\mathrm{f}}=10^{-2} ; \mathrm{n}_{\mathrm{z}}=10 ; \mathrm{n}_{\mathrm{t}}= \\
10 ; \mathrm{t}_{\mathrm{z}}=8760 \mathrm{~h} / \mathrm{ano}
\end{array} \\
& \mathrm{L}_{\mathrm{B}}=0,5 \times 10^{-3} \times 1 \times 10^{-2} \times\left(\frac{10}{10}\right) \times\left(8760 / 8760=5 \times 10^{-6}\right.
\end{aligned}
$$

Assim, pode-se calcular o valor de $\mathrm{R}_{\mathrm{B}}$ :

$$
R_{B}=0,038 \times 1 \times\left(5 \times 10^{-6}\right)=0,19 \times 10^{-6}
$$

Análise dos cálculos dos componentes de risco devido a descargas atmosféricas em uma linha energia conectada a uma estrutura (S3).

1 - Componente relativo ao tipo de ferimentos causados aos seres vivos por meio de choque elétrico $\left(\mathrm{R}_{\mathrm{u}}\right)$, apresentado no Quadro 2.

A Equação 11 apresentada no anexo A da ABNT NBR 5419-2 (2015), indica o número de eventos perigosos para a estrutura $\mathrm{N}_{\mathrm{L}}$.

$N_{L}=N_{G} \times A_{L} \times C_{I} \times C_{E} \times C_{T} \times 10^{-6}$

Ao analisar esta equação foi observado que $\mathrm{N}_{\mathrm{G}}$ já é conhecido.

De acordo com a norma ABNT NBR 5419-2 (2015), os parâmetros fator de instalação da linha $\left(C_{\mathrm{T}}\right)$, e o fator ambiental $\left(\mathrm{C}_{\mathrm{E}}\right)$ serão especificados através das Tabelas A.2 e A.4, respectivamente. Outro fator a seranalisado é a área de exposição equivalente de descargas atmosféricas que atingem a linha $\mathrm{em}^{2}$, dada através da equação:

$\mathrm{N}_{\mathrm{G}}=14,14 \mathrm{~km}^{2} / \mathrm{ano} ; \mathrm{C}_{\mathrm{I}}=0,5 ; \mathrm{C}_{\mathrm{E}}=0,1 ; \mathrm{C}_{\mathrm{T}}=1$

A Equação 12 apresentada no anexo $\mathrm{A}$ da ABNT NBR 5419-2 (2015), indica aárea de exposição equivalente $\mathrm{AL}$ :

$$
\mathrm{A}_{\mathrm{L}}=40 \times \mathrm{L}_{\mathrm{L}} \mathrm{m}^{2}
$$

O comprimento da linha (LL), é igual a 10 metros, com isso:

$$
A_{L}=40 \times 10=400 \mathrm{~m}^{2}
$$

Assim, é possível calcular o valor de $\mathrm{N}_{\mathrm{L}}$ :

$\mathrm{N}_{\mathrm{L}}=14,14 \times 400 \times 1 \times 0,1 \times 1 \times 10^{-6}=565,6 \times 10^{-6}$

A Equação 13 apresentada no anexo A da ABNT
NBR 5419-2 (2015), indica o cálculo de uma descarga atmosférica em uma linha causar ferimentos a seres vivos por meio de choque elétrico $\mathrm{Pu}$.

$$
\mathrm{P}_{\mathrm{U}}=\mathrm{P}_{\mathrm{TU}} \times \mathrm{P}_{\mathrm{EB}} \times \mathrm{P}_{\mathrm{LD}} \times \mathrm{C}_{\mathrm{LD}}
$$

De acordo com a norma ABNT NBR 5419-2 (2015), o valor da probabilidade $\left(\mathrm{P}_{\mathrm{TU}}\right)$ onde não há nenhuma medida de proteção será especificado através das Tabelas B.6, o valor da probabilidade $\left(\mathrm{P}_{\mathrm{EB}}\right)$ sem DPS será especificado através da Tabelas B.7, o valor da probabilidade (PLD) sabendo que a tensão suportável de impulso é de $2,5 \mathrm{kV}$ e que a linha de energia é aérea e não blindada e valor do fator dependente da blindagem do aterramento e isolação da linha será especificado através da tabela (B.4).

$$
\begin{aligned}
& \mathrm{P}_{\mathrm{TU}}=1 ; \mathrm{P}_{\mathrm{EB}}=1 ; \mathrm{P}_{\mathrm{LD}}=1 ; \mathrm{C}_{\mathrm{LD}}=1 ; \\
& \mathrm{P}_{\mathrm{U}}=1 \times 1 \times 1 \times 1=1
\end{aligned}
$$

A Equação 14 apresentada no anexo c da ABNT NBR 5419-2 (2015), indica os valores da perda $\mathrm{L}_{\mathrm{U}}$.

$$
\mathrm{Lu}=\mathrm{r}_{\mathrm{t}} \times \mathrm{L}_{\mathrm{T}} \mathrm{x}\left(\frac{n \mathrm{Z}}{n t}\right) \times \mathrm{t}_{\mathrm{z}} / 8760
$$

De acordo com a norma ABNT NBR 5419-2 (2015), o fator de redução da perda de vida humana dependendo do solo/piso $\left(\mathrm{r}_{\mathrm{t}}\right)$ deve ser especificado através da Tabela C.3 e o número relativo médio típico de vítimas feridas por choque elétrico devido a evento perigoso $\left(\mathrm{L}_{\mathrm{T}}\right)$ deve ser especificado através da Tabela C.2. O número de pessoas na zona $\left(\mathrm{n}_{\mathrm{z}}\right)$, o tempo durante o qual as pessoas estão presentes na zona expresso em $\mathrm{h} /$ ano $\left(\mathrm{t}_{\mathrm{z}}\right)$ e o número total de pessoas na estrutura $\left(\mathrm{n}_{\mathrm{t}}\right)$ são dados do projeto.

$$
\mathrm{r}_{\mathrm{t}}=10^{-3} ; \mathrm{L}_{\mathrm{T}}=10^{-2} ; \mathrm{n}_{\mathrm{z}}=10 ; \mathrm{n}_{\mathrm{t}}=10 ; \mathrm{t}_{\mathrm{z}}=8760 \mathrm{~h} / \text { ano }
$$

$$
L_{U}=10^{-3} \times 10^{-2} \times\left(\frac{10}{10}\right) \times(8760 / 8760)=10^{-5}
$$

Assim, pode-se calcular o valor de $\mathrm{R}_{\mathrm{U}}$ : adjacente.

Observação: $\mathrm{N}_{\mathrm{DJ}}=0$, pois não há estrutura

$$
\begin{aligned}
& \mathrm{R}_{\mathrm{U}}=\left(565,6 \times 10^{-6}+0\right) \times 1 \times 10^{-5} \\
& \mathrm{R}_{\mathrm{U}}=565,6 \times 10^{-10}
\end{aligned}
$$

2- Componente relativo ao tipo de ferimentos causados aos seres vivos por meio de choque elétrico $\left(\mathrm{R}_{\mathrm{V}}\right)$, apresentado no Quadro 2.

$\mathrm{N}_{\mathrm{L}}$ já foi calculado tendo o valor $\mathrm{N}_{\mathrm{L}}=565,6 \times 10^{-6}$ 
$\mathrm{N}_{\mathrm{DJ}}=0$, pois não há estrutura adjacente.

A Equação 15 apresentada no anexo A da ABNT NBR 5419-2 (2015), indica o cálculo de uma descarga atmosférica em uma linha causar anomalias físicas PV.

$$
\mathrm{P}_{\mathrm{V}}=\mathrm{P}_{\mathrm{EB}} \times \mathrm{P}_{\mathrm{LD}} \times \mathrm{C}_{\mathrm{LD}}
$$

Considerando os valores de $\mathrm{P}_{E B}, \mathrm{P}_{\mathrm{LD}}$ e $\mathrm{C}_{\mathrm{LD}}$ adotados para o cálculo de $\mathrm{P}_{\mathrm{U}}$ calculada anteriormente, tem-se:

$$
\begin{aligned}
& \mathrm{P}_{\mathrm{TU}}=1 ; \mathrm{P}_{\mathrm{EB}}=1 ; \mathrm{P}_{\mathrm{LD}}=1 ; \mathrm{C}_{\mathrm{LD}}=1 ; \\
& \mathrm{P}_{\mathrm{V}}=1 \times 1 \times 1=1
\end{aligned}
$$

A Equação 16 apresentada no anexo $\mathrm{c}$ da $\mathrm{ABNT}$ NBR 5419-2 (2015), indica os valores da perda $\mathrm{L}_{\mathrm{v}}$.

$$
\mathrm{L}_{\mathrm{B}}=\mathrm{r}_{\mathrm{p}} \times \mathrm{r}_{\mathrm{f}} \times \mathrm{h}_{\mathrm{Z}} \times \mathrm{L}_{\mathrm{F}} \mathrm{x}\left(\frac{n \mathrm{Z}}{n t}\right) \times\left(\mathrm{t}_{\mathrm{z}} / 8760\right)
$$

De acordo com a norma ABNT NBR 5419-2 (2015) o valor de $\mathrm{L}_{\mathrm{V}}=\mathrm{L}_{\mathrm{B}}$ então o valor de $\mathrm{L}_{\mathrm{V}}=5 \mathrm{x}$ $10^{-6}$.

Assim, pode-se calcular o valor de $\mathrm{R}_{\mathrm{v}}$ :

$$
\mathrm{R}_{\mathrm{v}}=\left(565,6 \times 10^{-6}+0\right) \times 1 \times\left(5 \times 10^{-6}\right)=3 \times 10^{-9}
$$

Para avaliação do risco (R1) para a zona predial em questão deve-se usar a equação apresentada no Quadro 4.

Os valores calculados foram:

$\mathrm{R}_{\mathrm{A}}=0,038 \times 10^{-5} ; \mathrm{R}_{\mathrm{B}}=0,19 \times 10^{-6} ; \mathrm{R}_{\mathrm{U}}=565,5 \times 10^{-10}$; $\mathrm{R}_{\mathrm{V}}=3 \times 10^{-10}$;

$\mathrm{R}_{1}=0,038 \times 10^{-6}+0,19 \times 10^{-6}+565,5 \times 10^{-10}+3 \times 10^{-10}=$ $0,63 \times 10^{-6}$

Comparando Risco tolerável $\left(\mathrm{R}_{\mathrm{T}}\right)$ para perda L1:

$$
\mathrm{R}<\mathrm{RT}
$$

$0,63 \times 10^{-6}<10^{-5}$

Levando-se em consideração que o risco encontrado é menor que o risco tolerável, através desta análise chega-se à conclusão que o prédio não necessita de implementação de um Sistema de Proteção contra Descarga Atmosférica.

\section{Conclusões}

Este trabalho apresentou a Análise de risco para determinação da necessidade da implementação de um Sistema de Proteção Contra descargas atmosféricas em uma construção localizada do município de Vassouras no estado do Rio de Janeiro em prol da segurança do imóvel e dos seres vivos que habitam o local.

O tema proposto é uma forma para que engenheiros de segurança do trabalho e engenheiros eletricistas tenham um entendimento inicial na forma de calcular os riscos que uma descarga atmosférica pode causar.

Foi analisado o método de avaliação dos principais requisitos necessários para o gerenciamento de risco como tipo de estrutura, as linhas conectadas na estrutura e o tipo de descarga como direta sendo aquela que incide diretamente na estrutura ou linha conectada à estrutura ou indireta sendo aquela que incide perto da estrutura ou perto da linha conectada à estrutura.

Os cálculos apresentados são uma previa de um análise em uma estrutura, contudo, a leitura da norma ABNT NBR 5419-2 (2015), se faz necessário, pois alguns requisitos que não eram aplicáveis ou pertinentes ao estudo de caso em questãonão foram abordados nesse artigo.

Torna-se importante salientar que apesar de o prédio analisado em questão não necessitar de um SPDA, a metodologia empregada neste estudo serve como um importante guia para profissionais da área no que se refere a aplicabilidade da ABNT NBR 5419-2 (2015).

\section{Referências}

ABNT - ASSOCIAÇÃO BRASILEIRA DE NORMAS TÉCNICAS. NBR 5419-2: Proteção de estruturas contra descargas atmosféricas. Rio de janeiro, 2015 .

Fergutz, Marcos.(2015).Sistema de Proteção Contra Descargas Atmosféricas SPDA, NBR 5419-2015: Princípios Gerais. Disponível em: <http://www. joinville.udesc.br/portal/professores/fergutz/materiais/spdal_v8_15.pdf >. Acesso em 06/maio/2017

Henriques, Marcos Ejczis.(2015).Estudo de caso: avaliação do sistema de proteção contra descargas atmosféricas de um barracão comercial situado na cidade de Curitiba. 2015. 40 f. Trabalho de Conclusão de Curso (Especialização) - Universidade Tecnológica Federal do Paraná, Curitiba.

Mamede Filho, João.(2017).Instalações elétricas Industriais: de acordo com a norma brasileiraNBR 2419/2015/ João Mamede Filho. - $9^{\circ}$ edição - Rio de Janeiro: LTC.

Souza, I.F.M .(2015). Estudo da norma ABNT5419/2015 com estudo de caso no antigo prédio do cetro de pesquisas físicas. Projeto de graduação apresentado ao curso de Engenharia Elétrica da Escola Politécnica da Universidade Federal do Rio de Janeiro, UFRJ, Rio de Janeiro - RJ.

Sueta, Hélio Eiji. (2015). ABNT NBR 5419 - 2 / 2015 GERENCIAMENTO DE RISCO. Disponível em: <http://abracopel.org/wp-content/ uploads/2015/09/Sueta.pdf>. Acesso em: 08 maio 2017.

Website do Instituto Nacional de Pesquisas Espacial - INPE. Disponível em: http://www.inpe.br/webelat/homepage/- Acesso em 05/05/2017

Portal o setor elétrico - Disponível em: http://www.osetoreletrico.com br/2016/2015/03/30/o-gerenciamento-de-risco-segundo-a-parte-2-da-abntnbr-5419/- Acesso em 20/04/2017 